\title{
Assessment of Diet Diversity and Eating Pattern of Undergraduate Students: A Pan India Study
}

\author{
Abhishek Kumar, Arshad Ayub*, Ria Roy, Aradhna Rai, Bhini Ameta, Ashmi Latheef, Aradhna Rani, Aysha, Ashmil \\ Muhammad Ali, Azad, Ashtami, Aradhna Rani, Avinash Pathak, Shamshad Ahmad, Pragya Kumar
}

\begin{abstract}
Abhishek Kumar, Arshad Ayub*, Ria Roy, Aradhna Rai, Bhini Ameta, Ashmi Latheef, Aradhna Rani, Aysha M, Ashmil Muhammad Ali, Azad AV, Ashtami $\mathrm{KH}$, Aradhna Rani, Avinash Pathak, Shamshad Ahmad, Pragya Kumar

Department of Community and Family Medicine, AlIMS Patna, Bihar, INDIA.
\end{abstract}

\section{Correspondence \\ Dr. Arshad Ayub \\ Senior Resident, Department of \\ Community and Family Medicine, AlIMS \\ Patna-801507, Bihar, INDIA. \\ Mobile no: +91 6202263099 \\ Email: drarshadayubcommed@gmail. \\ com \\ History \\ - Submission Date: 24-01-2020 \\ - Revised Date: 04-03-2020 \\ - Accepted Date: 19-04-2020}

DOI : 10.5530/ijmedph.2020.2.13

Article Available online

http://www.ijmedph.org/v10/i2

\section{Copyright}

(C) 2020 Phcog.Net. This is an openaccess article distributed under the terms of the Creative Commons Attribution 4.0 International license.

\begin{abstract}
Introduction: Dietary diversity has been universally identified as a key element of high quality diets. The tendency among undergraduate students with poor dietary patterns is high, due to lack of parental supervision, incorrect knowledge regarding food habits, stress of academic, professional and social life. This study was carried out to assess the diet diversity and eating pattern of undergraduate students of various colleges in India. Methods: This was a descriptive cross-sectional study conducted among 3046 undergraduate students across India by filling the online questionnaire shared through social media. The data on dietary diversity was collected by asking the number of consumed food groups in previous $24 \mathrm{hr}$ from the total food groups described by FAO, with anthropometric measurements. Data analysis was done using SPSS version 21. Results: Mean age of participants was $20.7 \pm 1.9$ years, the majority being females. About $42.2 \%$ student's skipped breakfast 1-2 times per week and another $28.5 \%$ skipped 3 or more times. About 34.7\% had poor DDS (Dietary Diversity Score) whereas 28.5\% had good DDS. Higher DDS mean was found in males (3.67) than females (3.23), overall mean being 3.4. Among 869 people with good DDS, $63.1 \%$ had normal BMI and $57.1 \%$ availed their meals from mess. There is significant distribution of DDS among different regions $(p=0.0007)$, graduation streams $(p=0.0014)$ and source of meals $(p<0.001)$. Conclusion: In this study, the diet diversity of about three-fourth participants was inadequate, showing their improper habit of food consumption and results also indicated their unhealthy eating pattern. Adequate dietary practice is recommended. Key words: Diet diversity, Eating pattern, Undergraduate students, Anthropometric measurement, BMI.
\end{abstract}

\section{INTRODUCTION}

Dietary diversity (DD) has been universally identified as a key element of high quality diets. As dietary factors are associated with increased risk of chronic diseases and under-nutrition, local and international dietary guidelines recommend to improve the diversity of the diet. ${ }^{1}$ Dietary Diversity has been defined as "the number of foods or food groups consumed over a given reference period." Dietary Diversity Score (DDS) has been considered as an indicator of diet quality associated inversely with risk of chronic diseases. ${ }^{3}$ The different food groups consumed and the pattern of diet hint to an individual's nutritional status. The analysis of dietary pattern gives a more comprehensive impression of the food consumption habits within a population. ${ }^{4}$ Nutritional status is the sum total of an individual's anthropometric indices as influenced by intake and utilization of nutrients, which is determined from information obtained by physical, biochemical and dietary studies. It is a result of interrelated factors influenced by quality and quantity of food consumed and the physical health of an individual. ${ }^{4}$ In this study, undergraduate students from colleges across
India are taken into consideration and their dietary habits and diet diversity is assessed. The assessment of Diet Diversity Score is done via $24 \mathrm{hr}$ Recall Method for the consumed food groups out of the total food groups classified according to the Food and Agricultural Organisation (FAO) for individual dietary diversity which include foods such as cereals, pulses, milk and milk products, meat, poultry and fish, green leafy vegetables, other vegetables, nuts and seeds, fats and oils, Vitamin A rich and other fruits. ${ }^{5}$

Undergraduate students encompass a substantial portion of the total population, that are likely to make poor judgement regarding their dietary habits. Tendency for consumption of unhealthy foods and poor dietary patterns is high, greatly due to lack of parental supervision, inadequate or incorrect knowledge regarding food habits and due to the stress of academic and professional as well as social life. ${ }^{6}$ Patterns of nutritional behaviours adopted in childhood and adolescents are mostly continued in adult life and hence their assessment becomes important to understand the risk for developing chronic diseases in the future. This study aims to
Cite this article: Kumar A, Ayub A, Roy R, Rai A, Ameta B, Latheef A, Rani A, Aysha M, Ali AM, Azad AV, Ashtami KH, Rani A, Pathak A, Ahmad S, Kumar P. Assessment of Diet Diversity and Eating Pattern of Undergraduate Students: A Pan India Study. Int J Med Public Health. 2020;10(2):58-63. 
assess the diet diversity and diet diversity score (DDS) of undergraduate college students using $24 \mathrm{hr}$ recall method and also to assess the nutritional status and pattern of diet of undergraduate college students.

\section{MATERIALS AND METHODS}

- Study design: This was a descriptive cross-sectional study.

- Study area: Colleges offering undergraduate courses (MBBS, BDS, B.E./B. Tech, B.Sc., B.A., LLB, B. Commerce and Others) across India were invited to be part of this study.

- Study setting: Department of Community and Family Medicine, AIIMS, Patna

- Study Duration: 3 weeks (21 days)

- Study Population: Undergraduate students in different colleges across India

- Exclusion criteria: Students who did not give consent for the study were excluded.

- Sample size determination: A pilot study was done among undergraduate students of 2017 Batch, AIIMS, Patna and it was found that $30 \%$ of students had good diet diversity (Diet diversity score $\geq 5$ ).

- Now, taking $p=0.3, q=(1-p)=0.7$ and allowable error as $2 \%$ we calculate a sample size using Taylor's formula $n=4 \mathrm{pq} / \mathrm{l}^{\star} \mathrm{l}$. Using this formula we got a sample size of 2100 .

- Sampling Technique: This study used a convenience sampling method.

- Data collection method: Online filling of the questionnaires was done by the undergraduate students from colleges across India via sharing the link to known personal contacts as well as contacts shared by the faculty in-charge through social networks such as WhatsApp, Instagram and Facebook. Dietary habits are observed by asking certain critical questions and the dietary diversity is assessed by the number of consumed food groups in the previous $24 \mathrm{hrs}$ from the total food groups as described by FAO.

- Study Tool: A pre-tested semi structured questionnaire was developed using google forms for the purpose of data collection, with a consent form attached along with it. The questionnaire included questions about general information, information concerning diet diversity and pattern and information regarding anthropometric measurements.

- a) General Information: Age, Gender, Educational Stream, Location of College (state or union territory), Type of Institution and Place of Current Residence (Hostel, Home etc.)

- b) Questions on diet diversity and pattern: Type of Diet, Number of major meals in a day, usual source of meals, timely consumption of meals, Skipping breakfast, snacking habits, consumption of the different food groups in last $24 \mathrm{hr}$.

- c) Anthropometric measurements as given by the participants: weight (in $\mathrm{kg}$ ) and height (in $\mathrm{cm}$ ).

- Food groups used in dietary diversity assessment: Ten food groups were used to assess the diet diversity score (DDS). The food groups were as. DDS Score was grouped into categories as Good (DDS $\geq 5$ ), Medium (DDS=3-4) and Low (DDS $\leq 2$ ).

- For the purpose of analysis, certain categories of data were clubbed together into groups:

- A) In the category of location of college, data across India was grouped region wise into North India (Uttar Pradesh, Uttarakhand, Haryana, Punjab, Delhi and Jammu and Kashmir), East India (Bihar, Jharkhand, West Bengal and Odisha), North-East (Arunachal Pradesh, Assam, Manipur, Meghalaya, Mizoram, Nagaland, Sikkim and Tripura), West India (Maharashtra, Rajasthan, Gujarat and Goa), South India (Andhra Pradesh, Telangana, Karnataka, Kerala, Tamil Nadu and Pondicherry) and Central India (Madhya Pradesh and Chhattisgarh).

- B) Consumption of food for short hunger pangs was grouped into

Fast Food and Beverages (junk/fast food, tea/coffee, soft drinks)

Fruits (Fruits, Dry Fruits)

Self- Prepared (Maggi, bread and butter, poha, food from home)

Biscuits and Chocolates

According to availability (as specified by the respondent) and

Remain hungry (includes drinking water).

- Data cleaning and Statistical Analysis: Templates were generated in Excel Sheets and data analysis was done using free online platform www.openepi.com (to determine $p$-value using $t$-test and ANOVA) and www.socscistatistics.com (to determine chi square). Statistical significance was set at $p<0.05$.

- Ethical approval: Declaration of Helsinki had been followed throughout the research work.

\section{RESULTS}

Mean age of participants was $20.7 \pm 1.9$ years, the majority being females. About $42.2 \%$ student's skipped breakfast 1-2 times per week and another $28.5 \%$ skipped 3 or more times. About $34.7 \%$ had poor DDS (Dietary Diversity Score) whereas $28.5 \%$ had good DDS. Higher DDS mean was found in males (3.67) than females (3.23), overall mean being 3.4. Among 869 people with good DDS, $63.1 \%$ had normal BMI and 57.1\% availed their meals from mess. There is significant distribution of DDS among different regions $(p=0.0007)$, graduation streams $(p=0.0014)$ and source of meals $(p<0.001)$. General information of the study participants have been described in Table 1 and their distribution across different regions of India have been shown in Figure 1. Figure 2 depicts a pie-chart depicting distribution of participants according to the usual source of meal. Diet pattern of the study participants have been shown in Table 2 . BMI category and habit of skipping breakfast of the study participants have been depicted in Figure 3 and 4 respectively. A pie-chart showing distribution of DDS category among the study participants have been depicted in Figure 5. Mean Diet Diversity Score (DDS) and Standard Deviation among all study participants have been explained in Table 3. Distribution of Mean DDS and Standard Deviation across gender using unpaired t-test have been explained in Table 4. Distribution of mean DDS among various undergraduate streams have been explained by Table 5 . Distribution of Mean DDS and Standard Deviation across various under graduate streams using ANOVA is depicted in Table 6. Association of DDS Category with different BMI classes and usual source of meal among the study participants have been explained in Table 7 and 8.

\section{DISCUSSION}

Poor eating habits is a major public health concern among young adults who transition into college or university life, when they are exposed to stress and lack of time. These factors pose an obstacle against the adoption of healthy behaviours and can therefore result in poor eating habits. Our study aimed to assess the diet diversity, eating pattern and DDS of undergraduate students using $24 \mathrm{hr}$ recall method and finding their nutritional status through BMI status. The mean DDS score was found to be 3.4, which was less than the good DDS category of 5 and above. 1057 (34.7\%) had poor DDS (Dietary Diversity Score) whereas 868 (28.5\%) had good DDS. Higher DDS mean was found in males (3.67) than females (3.23). $62.2 \%$ of the participants had normal BMI, 
$20.2 \%$ were overweight, $5.3 \%$ were obese and $12.3 \%$ were underweight, according to WHO criteria for BMI. There was significant distribution of DDS among different regions $(p=0.0007)$, graduation streams $(p=0.0014)$ and source of meals $(p<0.001)$. All these can be affected by many sociodemographic and psychological factors which have been examined in a study by Ganasegran et al. conducted in a Malaysian university, where it

Table 1: General information of the study participants ( $n=3046)$.

\begin{tabular}{|cccc|}
\hline Variable & Category & Frequency & Percentage (\%) \\
Gender & Female & 1609 & 52.8 \\
& Male & 1437 & 47.2 \\
Type of & Government & 2030 & 66.6 \\
Institution & Private & 1016 & 33.4 \\
Stream & MBBS & 2081 & 68.3 \\
& BDS & 137 & 4.5 \\
& B.A & 57 & 1.9 \\
& B. Commerce & 35 & 1.1 \\
& B.E./ B. Tech. & 299 & 9.8 \\
& LLB & 26 & 0.9 \\
& B. Sc. & 197 & 6.5 \\
& Others* & 214 & 7 \\
Currently & Hostel & 1980 & 65 \\
Residing In & Paying guest & 132 & 4.3 \\
& Home & 725 & 23.8 \\
& Rented Flats & 209 & 6.9
\end{tabular}

${ }^{*}$ Others include the streams of BCA, BBA and those not specified by the respondent.

Table 2: Diet pattern of the study participants $(n=3046)$.

\begin{tabular}{|c|c|c|c|}
\hline Variable & Category & Frequency & Percentage (\%) \\
\hline \multirow[t]{3}{*}{ Type of diet } & Vegetarian & 618 & 20.3 \\
\hline & Non- vegetarian & 2095 & 68.8 \\
\hline & Eggetarian & 333 & 10.9 \\
\hline \multirow{4}{*}{$\begin{array}{l}\text { No. of major } \\
\text { meals taken in } \\
\text { a day }\end{array}$} & 1 & 102 & 3.3 \\
\hline & 2 & 1124 & 36.9 \\
\hline & 3 & 1661 & 54.6 \\
\hline & $>3$ & 159 & 5.2 \\
\hline \multirow{5}{*}{$\begin{array}{l}\text { Number of times } \\
\text { taking snacks in } \\
\text { a day }\end{array}$} & 0 & 251 & 8.2 \\
\hline & 1 & 1466 & 48.1 \\
\hline & 2 & 1055 & 34.6 \\
\hline & 3 & 209 & 6.9 \\
\hline & $>3$ & 65 & 2.2 \\
\hline \multirow{6}{*}{$\begin{array}{l}\text { Tackling of short } \\
\text { term hunger }\end{array}$} & Remain hungry & 373 & 12.3 \\
\hline & $\begin{array}{l}\text { According to } \\
\text { availability }\end{array}$ & 746 & 24.5 \\
\hline & Self- prepared & 128 & 4.2 \\
\hline & $\begin{array}{c}\text { Fast food and } \\
\text { beverages }\end{array}$ & 1204 & 39.5 \\
\hline & $\begin{array}{l}\text { Chocolates and } \\
\text { biscuits }\end{array}$ & 387 & 12.7 \\
\hline & Fruits & 208 & 6.8 \\
\hline
\end{tabular}

was seen that there existed significant associations between poor eating habits and age, smoking and drinking behaviours, loneliness and being upset. It was found that $43.9 \%$ of the respondents had breakfast daily, ${ }^{7}$ in comparison to our study where only $29.3 \%$ had a daily breakfast and $28.5 \%$ skipped it thrice or more every week.

This pattern was also found to be consistent with a UK based crosssectional study where about $30 \%$ of the students said they skipped breakfast most days. In addition, the frequent consumption of snacks and light meals is a very common aspect of food behaviour among undergraduates. The low leisure-time physical activity $(p<0.001)$, University attendance $(p=0.003)$, full time student status $(p=0.001)$ and living with parents and relatives $(p<0.001)$ were independently associated significantly with higher 'snacking' pattern scores in the study. The 'snacking' pattern contribute to acquiring of the least nutrients and was positively correlated with energy intake and hence weight gain. ${ }^{8}$ Breakfast is considered the most important meal of the day as it is known to provide energy for the brain and improve learning. It also contributes significantly to the total daily energy and nutrient intake. Skipping breakfast may affect performance during the rest of the day. ${ }^{9}$

Table 3: Mean Diet Diversity Score (DDS) and Standard Deviation among all study participants.

\begin{tabular}{|cccc|}
\hline DDS & Frequency & Mean & SD \\
\hline Overall & 3046 & 3.4 & 1.9 \\
\hline
\end{tabular}

Table 4: Distribution of Mean DDS and Standard Deviation across gender using unpaired t-test.

$\begin{array}{ccccc}\text { Gender } & \text { Frequency } & \text { Mean } & \text { SD } & P \text {-value } \\ \text { Male } & 1437 & 3.67 & 2.02 & \\ \text { Female } & 1609 & 3.23 & 1.83 & 0.0001\end{array}$

Table 5: Distribution of Mean DDS and Standard Deviation across various under graduate streams using ANOVA.

\begin{tabular}{|ccccc|}
\hline Stream & Frequency & Mean & SD & $P$-value \\
MBBS & 2081 & 3.61 & 1.93 & \\
BDS & 137 & 3.04 & 1.86 & \\
B.E./ B. Tech. & 299 & 3.14 & 1.94 & \\
B. A. & 57 & 3.38 & 1.56 & \\
B. Commerce & 35 & 2.60 & 1.26 & 0.0014 \\
LLB & 26 & 3.42 & 1.47 & \\
B. Sc. & 197 & 2.88 & 1.71 & \\
Others & 214 & 3.08 & 2.05 & \\
\hline
\end{tabular}

Table 6: Distribution of Mean DDS and Standard Deviation across different regions of India using ANOVA.

\begin{tabular}{|ccccc|}
\hline Region & Frequency & Mean & SD & $p$-value \\
North India & 732 & 3.4 & 1.8 & \\
North East & 140 & 2.8 & 1.70 & \\
East India & 886 & 3.79 & 1.82 & \\
West India & 363 & 3.30 & 1.70 & 0.0007 \\
South India & 770 & 3.22 & 1.96 & \\
Central India & 155 & 3.55 & 1.54 & \\
\hline
\end{tabular}


Table 7: Association of DDS Category with different BMI classes among the study participants.

\begin{tabular}{|c|c|c|c|c|c|c|c|c|c|c|}
\hline DDS & & & & & Cate & ry of BM & & & & \\
\hline Category & Unde & eight (\%) & Nor & nal (\%) & Over & ight (\%) & & se (\%) & Total & $p$-value \\
\hline Good & 81 & $(21.6 \%)$ & 548 & $(28.9 \%)$ & 197 & $(31.9 \%)$ & 43 & $(26.3 \%)$ & 869 & \\
\hline Medium & 132 & $(35.2 \%)$ & 710 & (37.5\%) & 216 & $(35.0 \%)$ & 63 & $(38.6 \%)$ & 1121 & \\
\hline Low & 162 & (43.2\%) & 634 & (33.5\%) & 203 & (32.9\%) & 57 & $(34.9 \%)$ & 1056 & 0.003 \\
\hline Grand Total & 375 & $(100 \%)$ & 1892 & (100\%) & 616 & $(100 \%)$ & 163 & $(100 \%)$ & 3046 & \\
\hline
\end{tabular}

Table 8: Association of DDS Category with usual source of major meals among study participants.

\begin{tabular}{|c|c|c|c|c|c|c|c|c|c|c|}
\hline \multirow{3}{*}{$\begin{array}{c}\text { DDS } \\
\text { Category } \\
\text { Good }\end{array}$} & \multicolumn{10}{|c|}{ Usual source of meals } \\
\hline & \multicolumn{2}{|c|}{ Homemade (\%) } & \multicolumn{2}{|c|}{ Mess (\%) } & \multicolumn{2}{|c|}{$\begin{array}{l}\text { Order from } \\
\text { Outside (\%) }\end{array}$} & \multicolumn{2}{|c|}{$\begin{array}{c}\text { Self-prepared } \\
\text { (\%) }\end{array}$} & Total & \multirow[t]{2}{*}{$p$-value } \\
\hline & 288 & $(36.1 \%)$ & 496 & $(26.3 \%)$ & 46 & $(18.5 \%)$ & 39 & $(33.0 \%)$ & 869 & \\
\hline Medium & 234 & $(29.3 \%)$ & 745 & $(39.5 \%)$ & 104 & $(41.9 \%)$ & 38 & $(32.2 \%)$ & 1121 & \multirow{2}{*}{0.001} \\
\hline Low & 274 & $(34.4 \%)$ & 643 & $(34.1 \%)$ & 98 & $(39.5 \%)$ & 41 & $(34.7 \%)$ & 1056 & \\
\hline Grand total & 796 & $(100 \%)$ & 1884 & $(100 \%)$ & 248 & $(100 \%)$ & 118 & $(100 \%)$ & 3046 & \\
\hline
\end{tabular}

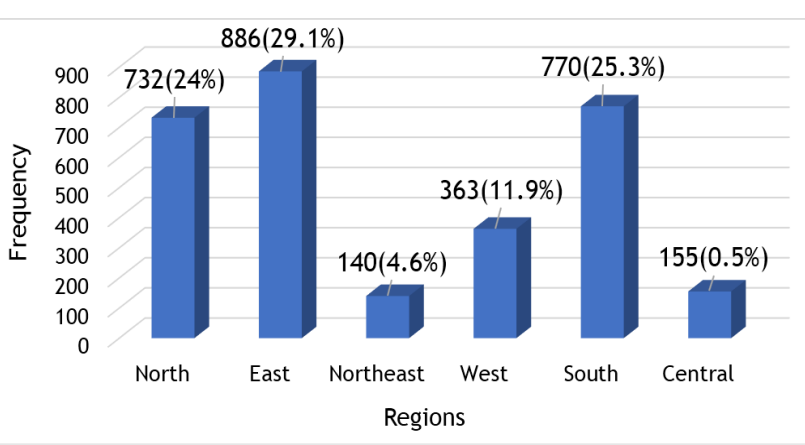

Figure 1: Distribution of participants across various regions of India.

In face to face interviews conducted in five universities in Ankara, Turkey, however it was found that breakfast and lunch were the most frequently skipped meals, with a total of $47.7 \%$ of students skipping breakfast and $25.2 \%$ skipping lunch. $25.1 \%$ for males and $5.6 \%$ for females were overweight. Also, $1.8 \%$ of male and $16.3 \%$ of female students were found to be underweight, probably because desire for a thin body size affects especially the nutritional status in women. Instead of DDS, nutritional intake was measured and it was found that $78.4 \%$ men and $81.1 \%$ women had energy deficiency, pointing towards poor nutritional status. ${ }^{10}$ In a cross sectional diet diversity study carried out in Ogun and Lagos states of South west Nigeria, $8 \%$ of the students were overweight and $3 \%$ were obese. The total dietary diversity score for male was $4.07 \pm$ 1.84 while female had $4.53 \pm 3.07$, the difference being significant and the score being done for 9 food groups. The DDS according to gender in our study however shows the opposite pattern, with males having higher mean DDS than females. Also 20.2\% were overweight, a much higher proportion than that of the Nigerian study, while in both studies majority of the students belonged to the normal BMI category according to the WHO criteria. ${ }^{11}$ In another Southern Nigerian study carried out

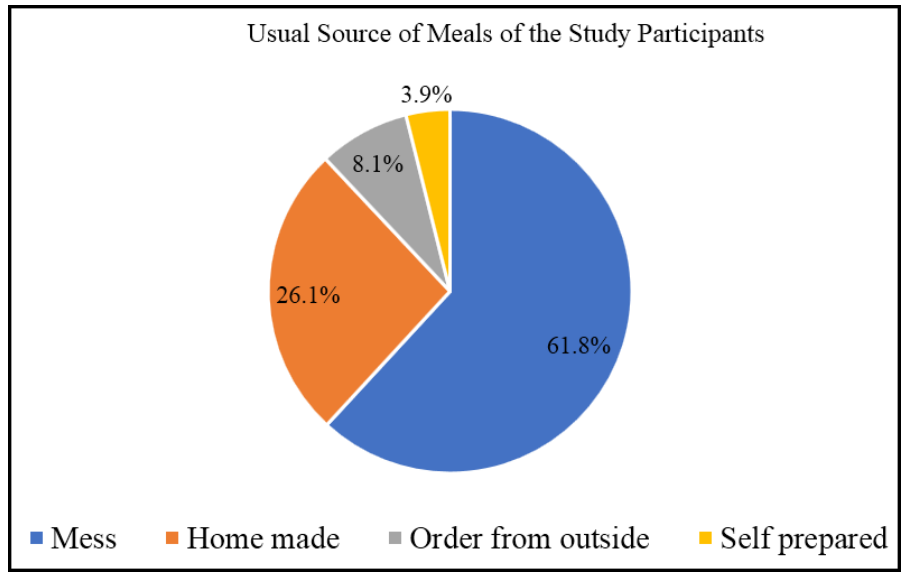

Figure 2: Pie-chart depicting distribution of participants according to the usual source of meal.

among undergraduate students in Igbinedion University, Okada, 49.0\% of the respondents had high dietary diversity score $(\geq 6)$ while $26.5 \%$ had low dietary diversity score $(\leq 3)$, among whom more males $(51.8 \%)$ had a significantly $(p<0.004)$ higher dietary diversity score compared to the females (47.0\%). ${ }^{4}$ A Chinese study, on the other hand, showed increasing diet diversity with rapid urbanization in the past decades. As DDS increased, consumption also increased excessively in most food groups like grains, meat and oil, while consumption of other six food groups including cereals and vegetables were inadequate. In this way, people with medium (5) and high ( $\geq 6)$ DDS ingested more energy than the recommended quantity. The eating pattern is not balanced. $25.2 \%$ of the respondents had low DDS score, which is lesser than that of our study $(34.7 \%){ }^{12}$ The diet diversity also varies across geographical regions, as is evident by the research carried out by Papadaki et al. at Athens Agricultural University in Greece, where a comparison has been done between students living at home and those who stay away from home. 


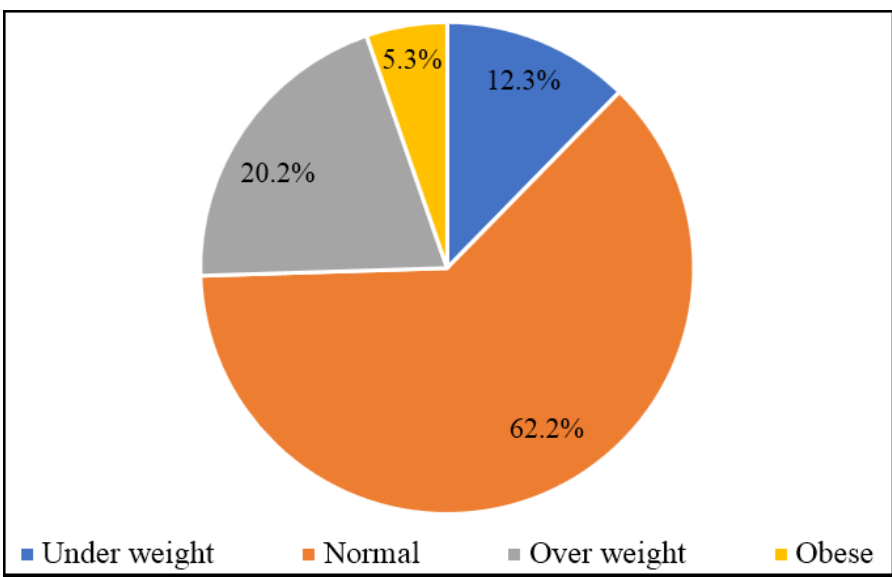

Figure 3: Pie-chart showing distribution of BMI category of study participants.

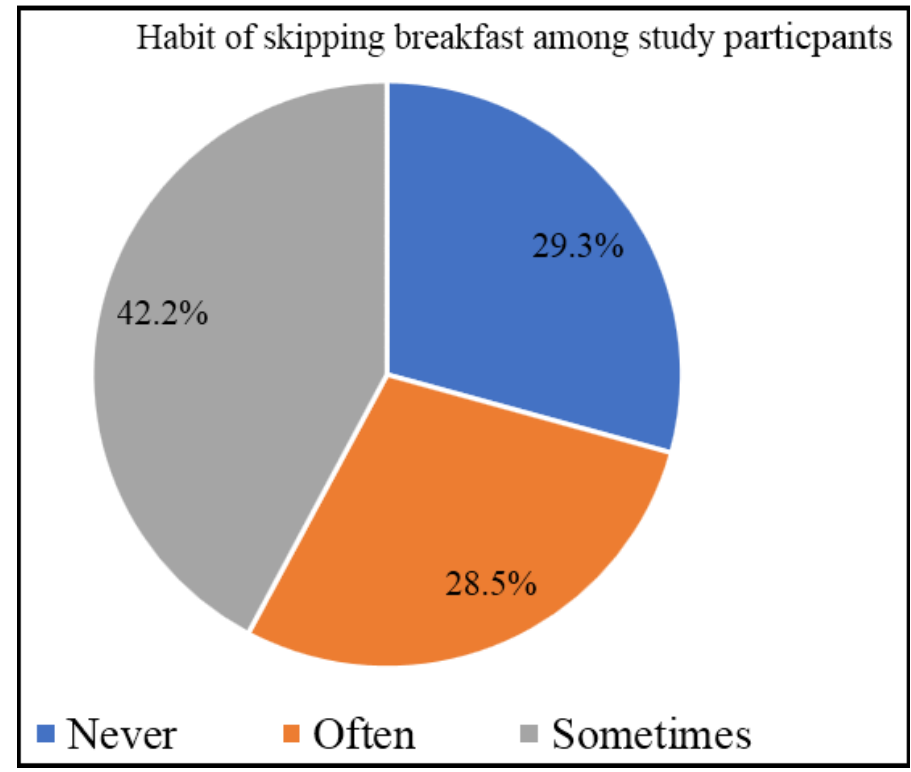

Figure 4: Pie chart showing habit of skipping breakfast among study participants.

Students at home did not show major changes in their eating habits since starting University. But students living away from home decreased their weekly consumption of fresh fruit, cooked and raw vegetables, oily fish, seafood, pulses and olive oil, which are readily available in these regions and increased their sugar, wine, alcohol and fast food intake, suggesting that shifting away from home and taking responsibility for food preparation and purchasing for the first time affect dietary habits of students, consequently showing significant difference of food habits and consumption during comparison between the groups. ${ }^{13}$ Such a comparison between the two groups and their association with dietary behaviour has not been done in our study.

It is evident that majority of diet diversity studies have been done outside India, however, similar to the previous study, interviews of 50 hostelite and 50 localite undergraduate medical students from Indira Gandhi Medical College (IGGMC), Nagpur were conducted to document both quantitative and qualitative dietary pattern among the students. From anthropometric measurements, it was calculated that $40 \%$ of students residing at hostel were underweight as compared to localites (10\%). Also, $6 \%$ hostelites were found to be overweight compared to $4 \%$ of

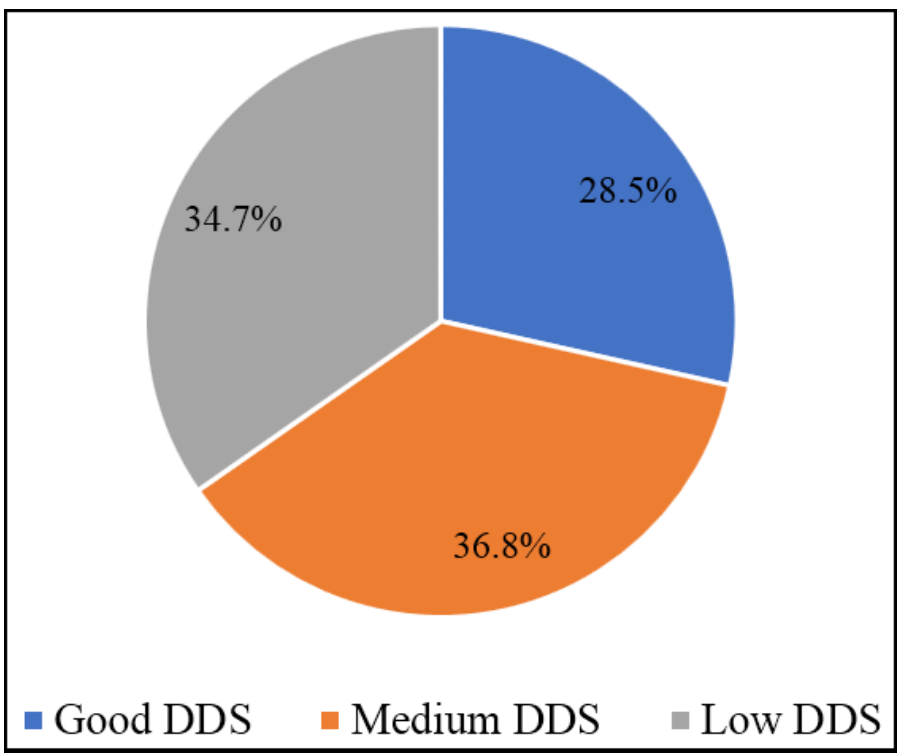

Figure 5: Pie-chart showing distribution of DDS category among the study participants.

localites. There was statistically significant difference found between the two groups with respect to frequency of consumption of junk foods. ${ }^{14}$ In another part of India, data on socio-demographic, lifestyle, exercise and dietary habits were collected from a college in Raichur due to the hypothesis that increase in the prevalence of overweight and obesity due to inappropriate diet and inactive lifestyle, over-consumption of calories, especially fast food, snacks and soft drinks leads to chronic diseases and about $80 \%$ of all heart disease, stroke, type 2 diabetes mellitus and $40 \%$ of cancer could be avoided through healthy diet and exercise. Majority (70\%) of the students skipped breakfast frequently, whereas $45.8 \%$ said they consumed junk food more than once in a week. Only 147 (38.3\%) out of 384 students walked at least for $30 \mathrm{~min}$ and did exercise daily. ${ }^{15} \mathrm{In}$ stark contrast to this, about $68 \%$ responders out of 130 said that they had breakfast daily in a diet pattern study at a health university in western Maharashtra, India. ${ }^{16}$ While consuming junk food and skipping breakfast has been extensively explored in our study, the duration of exercise done by the participants were not found, hence exercise, apart from food intake, acts as another factor affecting the BMI. Quality of food consumption is as important as quantity. There is increasing scientific evidence that adequate consumption of fruits and vegetables decreases the risk of major chronic diseases. The lack of nutrition literacy and the degree to which fast food are quickly becoming embedded in our society pave way towards various chronic health problems. The lack of information about food choices, nutritional information and its application, less availability to healthy foods and a range of cultural influences and socioeconomic considerations also decrease the chances for healthy eating and living. There is immense need for conveying the balanced food habits that the students can acquire from the start, to avoid the unhealthy patterns of nutritional behaviours which can continue well into adult life and risk increasing the burden of chronic illness in the society as well as on the health system.

\section{Limitation of the study}

We could not get responses from all the streams and all the states of India in our study. The responses we got are not having equal representation from different streams and states of India. Hence the scope of future research should be broadened to include a lager sample size of undergraduate students by including all the streams and different states of India, thus having equal representation to have a better picture of diet 
diversity and nutritional status. Also, anthropometry was asked from the students via recall method and not directly measured, which may have introduced recall bias in the study.

\section{CONCLUSION}

In this study it was found that mean age of the participants was 20.7 \pm 1.9 years. Mean DDS of the study participants was found to be 3.4 which is less than 5 (Good DDS), showing their improper habit of food consumption. In general, most of the students in this study had healthy eating habits, including 3 timely major meals and 1 snacks per day, except about $70 \%$ of total participants skipping breakfast at least once a week and about two-thirds eating fast food and beverages as their means of tackling short term hunger. Although students thought that their meal was adequate in quantity but they were not confident about its quality.

\section{ACKNOWLEDGEMENT}

We acknowledge the contribution and guidance of our faculty members, work of undergraduate students in data collection, analysis and results preparation in this project.

\section{CONFLICT OF INTEREST}

The authors declare none.

\section{ABBREVIATIONS}

ANOVA: Analysis of Variance; BMI: Body Mass Index; DDS: Diet Diversity Score; FAO: Food and Agriculture Organization; SPSS: Statistical Package for Social Sciences.

\section{REFERENCES}

1. Use of dietary diversity score as a proxy indicator of nutrient adequacy of rural elderly people in Sri Lanka. BMC Research Notes. 2012;5(1):469. [Cited 2019 Dec 15]. Available from: https://bmcresnotes.biomedcentral.com/articles/10.1186/1756-0500-5-469

2. Pangaribowo EH, Gerber N, Torero M. Food and Nutrition Security Indicators: A Review. University of Bonn, Center for Development Research (ZEF). 2013;1
49. [cited 2019 Dec 15]. (Working Papers). Report No.: 147911. Available from: https://ideas.repec.org/p/ags/ubonwp/147911.html

3. Nachvak SM, Abdollahzad H, Mostafai R, Moradi S, PasdarY, Rezaei M, et al. Dietary Diversity Score and Its Related Factors among Employees of Kermanshah University of Medical Sciences. Clin Nutr Res. 2017;6(4):247-55.

4. Omage K, Omuemu VO. Assessment of dietary pattern and nutritional status of undergraduate students in a private university in southern Nigeria. Food Sci Nutr. 2018;6(7):1890-7.

5. Household Dietary Diversity. Food and Agriculture Organization of the United Nations. 2019. [cited 2019 Dec 15]. Available from: http://www.fao.org/nutrition/ assessment/tools/household-dietary-diversity/en/

6. Kapka-Skrzypczak L, Bergier B, Diatczyk J, Niedźwiecka J, Biliński P, Wojtyła A Dietary habits and body image perception among Polish adolescents and young adults: A population based study. Ann Agric Environ Med. 2012;19(2):299-308.

7. Ganasegeran K, Al-Dubai SA, Qureshi AM, Al-abed AA, Rizal AM, Aljunid SM. Social and psychological factors affecting eating habits among university students in a Malaysian medical school: A cross-sectional study. Nutr J. 2012;11(1):48

8. Sprake EF, Russell JM, Cecil JE, Cooper RJ, Grabowski P, Pourshahidi LK, et al. Dietary patterns of university students in the UK: A cross-sectional study. Nutr J. 2018;17(1):90.

9. Ackuaku-Dogbe EM, Abaidoo B. Breakfast Eating Habits among Medical Students. Ghana Med J. 2014;48(2):66-70.

10. Neslişah R, Emine AY. Energy and nutrient intake and food patterns among Turkish university students. Nutr Res Pract. 2011;5(2):117-23

11. Akinlua OAJ. Dietary Diversity Score and Nutritional Status of Undergraduates in South West Nigeria. J Obes Weight Loss Ther. 2014;4:2. [cited 2019 Dec 15] Available from: http://www.omicsgroup.org/journals/dietary-diversity-scoreand-nutritional-status-of-undergraduates-in-south-west-nigerias-2165-7904.S4003.php?aid $=24829$

12. Zhang Q, Chen X, Liu Z, Varma DS, Wan R, Zhao S. Diet diversity and nutritional status among adults in southwest China. Plos One. 2017;12(2):e0172406.

13. Papadaki A, Hondros GA, Scott J, Kapsokefalou M. Eating habits of University students living at, or away from home in Greece. Appetite. 2007;49(1):169-76.

14. Khan B, Sukhsohale ND, Khamgaonkar MB. Nutritional Status and Dietary Pattern of Undergraduate Medical Students of Central India. Sch J App Med Sci. 2015;3(1A):49-52.

15. Baseer MD, Revathi, Ayesha SN, Ramesh, Hiremath SG, Sreekantha. Dietary Habits and Life Style among Pre-university College Students in Raichur, India. International Journal of Research in Health Sciences. 2015;3(3):407-11. [cited 2019 Dec 16]; Available from: http://ijrhs.org/article/2015/3/3-4

16. Vibhute N, Baad R, Belgaumi U, Kadashetti V, Bommanavar S, Kamate W. Dietary habits amongst medical students: An institution-based study. J Fam Med Prim Care. 2018:7(6):1464. 